\title{
Boundary Points and Resolution
}

\author{
Eugene Goldberg \\ eu.goldberg@gmail.com
}

\begin{abstract}
We use the notion of boundary points to study resolution proofs. Given a CNF formula $F$, a lit( $x$ )-boundary point is a complete assignment falsifying only clauses of $F$ having the same literal lit $(x)$ of variable $x$. A lit $(x)$-boundary point mandates a resolution on variable $x$. Adding the resolvent of this resolution to $F$ eliminates this boundary point. Any resolution proof has to eventually eliminate all boundary points of $F$. Hence one can study resolution proofs from the viewpoint of boundary point elimination. We use equivalence checking formulas to compare proofs of their unsatisfiability built by a conflict driven SAT-solver and very short proofs tailored to these formulas. We show experimentally that in contrast to proofs generated by this SAT-solver, almost every resolution of a specialized proof eliminates a boundary point. This implies that one may use the share of resolutions eliminating boundary points as a metric for proof quality.
\end{abstract}

Keywords: SAT-solver, boundary points, resolution, proof quality

\section{Introduction}

Resolution-based SAT-solvers $[4,9,13,16,18,19,20]$ have achieved great success in numerous applications. Importantly, in many cases, the quality of resolution proofs generated by a SAT-solver matters as much as its performance. For example, in bounded model checking [5], a resolution proof is used to identify the part of the circuit relevant to a particular property [7]. In interpolation based model checking [15], the size of interpolant strongly depends on that of the resolution proof it is extracted from. In [10], we showed that high-quality tests can be obtained from a resolution proof. The number of these tests is proportional to the number of resolutions in the proof. So proof reduction means getting a more compact test set.

In this paper, we study the relation between resolution and boundary points [12]. The motivation here is as follows. To show that a CNF formula is unsatisfiable it is sufficient to eliminate all its boundary points. Resolution can be viewed as a method of boundary point elimination. Relating resolution proofs with boundary point elimination, may lead to a) better understanding of resolution proofs; b) identifying proof redundancies; c) designing SAT-solvers that generate smaller proofs.

Given a CNF formula $F\left(\mathrm{x}_{1}, \ldots, x_{\mathrm{n}}\right)$, a non-satisfying complete assignment $\boldsymbol{p}$ is called a $\operatorname{lit}\left(x_{\mathrm{i}}\right)$-boundary point, if it falsifies only the clauses of $F$ that have literal $\operatorname{lit}\left(x_{\mathrm{i}}\right)$. The name is due to the fact that for satisfiable formulas the set of such points contain the boundary between satisfying and unsatisfying assignments. If $F$ is unsatisfiable, for 
every $\operatorname{lit}\left(x_{\mathrm{i}}\right)$-boundary point $\boldsymbol{p}$ there is a resolvent of two clauses of $F$ on variable $x_{\mathrm{i}}$ that eliminates $\boldsymbol{p}$ (i.e. after adding such a resolvent to $F, \boldsymbol{p}$ is not a boundary point anymore). On the contrary, for a non-empty satisfiable formula $F$, there is always a boundary point that can not be eliminated by adding a clause implied by $F$.

To prove that a CNF formula $F$ is unsatisfiable it is sufficient to eliminate all its boundary points. In the resolution proof system [3], one reaches this goal by adding to $F$ resolvents. If formula $F$ has a $\operatorname{lit}\left(x_{\mathrm{i}}\right)$-boundary point, a resolution proof has to have a resolution operation on variable $x_{\mathrm{i}}$. The resolvents of a resolution proof eventually eliminate all boundary points. (A formula with an empty clause does not have any boundary points.) However, as we show experimentally many resolution operations of proofs generated by a conflict driven SAT-solver do not eliminate any boundary points (non-boundary resolutions). We use the ratio of boundary and nonboundary resolutions of a proof as a redundancy measure called SBR metric (Share of Boundary Resolutions).

To check if there is a relation between the value of SBR metric and proof quality we computed the values of this metric for two kinds of proofs for equivalence checking formulas. (These formulas describe equivalence checking of two copies of a combinational circuit.) Namely, we considered short proofs of linear size particularly tailored for equivalence checking formulas and much longer proofs generated by a SAT-solver with conflict driven learning. We showed experimentally that the share of boundary resolution operations in high-quality specialized proofs is much greater than in proofs generated by the SAT-solver. This implies that the SATsolver's proofs may have some redundancies.

The contribution of this paper is threefold. First, we show that one can view resolution as elimination of boundary points. Second, we introduce the SBR metric that can be potentially used as a measure of proof redundancy. Third, we give some experimental results about the relation between SBR -metric and proof quality.

This paper is structured as follows. Section 2 introduces main definitions. Some properties of boundary points are given in Section 3. Section 4 views a resolution proof as a process of boundary point elimination. A class of equivalence checking formulas and their short resolution proofs are described in Section 5. Experimental results are given in Section 6. Some relevant background is recalled in Section 7. Conclusions and directions for future research are listed in Section 8.

\section{Basic Definitions}

Definition 1. A literal of a Boolean variable $x_{\mathrm{i}}$ (denoted as litt $\left.x_{\mathrm{i}}\right)$ ) is a Boolean function of $x_{\mathrm{i}}$. The identity function (denoted just as $x_{\mathrm{i}}$ ) is called the positive literal of $x_{\mathrm{i}}$. The negation function (denoted as $\sim x_{\mathrm{i}}$ ) is called the negative literal of $x_{\mathrm{i}}$.

Definition 2. A clause is the disjunction of literals where no two (or more) literals of the same variable can appear. A CNF formula is the conjunction of clauses. We will also view a CNF formula as a set of clauses. 
Definition 3. Given a CNF formula $F\left(x_{1}, . ., x_{\mathrm{n}}\right)$, a complete assignment (also called a point) is a mapping $\left\{x_{1}, . ., x_{\mathrm{n}}\right\} \rightarrow\{0,1\}$. Given a complete assignment $\boldsymbol{p}$ and a clause $C$, denote by $C(\boldsymbol{p})$ the value of $C$ when its variables are assigned by $\boldsymbol{p}$. A clause $C$ is satisfied (respectively falsified) by a complete assignment $\boldsymbol{p}$, if $C(\boldsymbol{p})=1$ (respectively $C(\boldsymbol{p})=0)$.

Definition 4. Given a CNF formula $F$, a satisfying assignment $\boldsymbol{p}$ is a complete assignment satisfying every clause of $F$. The satisfiability problem (SAT) is to find a satisfying assignment for $F$ or to prove that such an assignment does not exist.

Definition 5. Let $F$ be a CNF formula and $\boldsymbol{p}$ be a complete assignment. Denote by $\operatorname{Unsat}(\boldsymbol{p}, F)$ the set of all clauses of $F$ falsified by $\boldsymbol{p}$.

Definition 6. Given a CNF formula $F\left(x_{1}, . ., x_{n}\right)$, a complete assignment $\boldsymbol{p}$ is called a $\operatorname{lit}\left(x_{\mathrm{i}}\right)$-boundary point if $\operatorname{Unsat}(\boldsymbol{p}, F)$ is not empty and every clause of $\operatorname{Unsat}(\boldsymbol{p}, F)$ contains literal $\operatorname{lit}\left(x_{\mathrm{i}}\right)$.

Example 1. Let $F$ consist of 5 clauses: $C_{1}=x_{2}, C_{2}=\sim x_{2} \vee x_{3}, C_{3}=\sim x_{1} \vee \sim x_{3}$, $C_{4}=x_{1} \vee \sim x_{3}, C_{5}=\sim x_{2} \vee \sim x_{3}$. Complete assignment $\boldsymbol{p}_{1}=\left(x_{1}=0, x_{2}=0, x_{3}=1\right)$ falsifies clauses $C_{1}, C_{4}$. So $\operatorname{Unsat}\left(\boldsymbol{p}_{1}, F\right)=\left\{C_{1}, C_{4}\right\}$. There is no literal shared by the clauses of $\operatorname{Unsat}\left(\boldsymbol{p}_{1}, F\right)$. Hence $\boldsymbol{p}_{1}$ is not a boundary point. On the other hand, $\boldsymbol{p}_{2}=$ $\left(x_{1}=0, x_{2}=1, x_{3}=1\right)$ falsifies the clauses $C_{4}, C_{5}$ sharing literal $\sim x_{3}$. So $p_{2}$ is a $\sim x_{3}$-boundary point

\section{Basic Properties of Boundary Points}

In this section we give some properties of boundary points.

\subsection{Basic Propositions}

In this subsection, we prove the following propositions. The set of boundary points contains the boundary between satisfying and unsatisfying assignments (Proposition 1). A CNF formula without boundary points is unsatisfiable (Proposition 2). Boundary points come in pairs (Proposition 3).

Definition 7. Denote by Bnd pnts $(F)$ the set of all boundary points of a CNF formula $F$. We assume that a $\operatorname{lit}\left(x_{\mathrm{i}}\right)$-boundary point $\boldsymbol{p}$ is specified in $\operatorname{Bnd} \operatorname{pnts}(F)$ as the pair $\left(\operatorname{lit}\left(x_{\mathrm{i}}\right), \boldsymbol{p}\right)$. So the same point $\boldsymbol{p}$ may be present in $B n d$ pnts $(F)$ more than once (e.g. if $\boldsymbol{p}$ is a $\operatorname{lit}\left(x_{\mathrm{i}}\right)$-boundary point and a $\operatorname{lit}\left(x_{\mathrm{j}}\right)$-boundary point at the same time).

Proposition 1. Let $F$ be a satisfiable formula whose set of clauses is not empty. Let $\boldsymbol{p}_{\mathbf{1}}$ and $\boldsymbol{p}_{\mathbf{2}}$ be two complete assignments such that a) $F\left(\boldsymbol{p}_{\mathbf{1}}\right)=0, F\left(\boldsymbol{p}_{2}\right)=1$; b) $\boldsymbol{p}_{\mathbf{1}}$ and $\boldsymbol{p}_{\mathbf{2}}$ are different only in the value of variable $x_{\mathrm{i}}$. Then $\boldsymbol{p}_{1}$ is a lit $\left(x_{\mathrm{i}}\right)$-boundary point. 
Proof. Assume the contrary i.e. $\operatorname{Unsat}\left(\boldsymbol{p}_{1}, F\right)$ contains a clause $C$ of $F$ that does not have variable $x_{\mathrm{i}}$. Then $\boldsymbol{p}_{2}$ falsifies $C$ too and so $\boldsymbol{p}_{2}$ cannot be a satisfying assignment. A contradiction.

Proposition 1 means that the set Bnd pnts $(F)$ contains the boundary between satisfying and unsatisfying assignments of a satisfiable CNF formula $F$.

Proposition 2. Let $F$ be a $\mathrm{CNF}$ formula that has at least one clause. If Bnd_pnts $(F)=\varnothing$, then $F$ is unsatisfiable.

Proof. Assume the contrary i.e. Bnd pnts $(F)=\varnothing$ and $F$ is satisfiable. Since $F$ is not empty, one can always find two points $\boldsymbol{p}_{1}$ and $\boldsymbol{p}_{2}$ such that $F\left(\boldsymbol{p}_{1}\right)=0$ and $F\left(\boldsymbol{p}_{2}\right)=1$ and that are different only in the value of one variable $x_{\mathrm{i}}$ of $F$. Then according to Proposition $1, \boldsymbol{p}_{\mathbf{1}}$ is a $\operatorname{lit}\left(x_{\mathrm{i}}\right)$-boundary point. A contradiction.

Proposition 3. Let $\boldsymbol{p}_{\mathbf{1}}$ be a lit $\left(x_{\mathrm{i}}\right)$-boundary point for a CNF formula $F$. Let $\boldsymbol{p}_{\mathbf{2}}$ be the point obtained from $\boldsymbol{p}_{\mathbf{1}}$ by changing the value of $x_{\mathrm{i}}$. Then $\boldsymbol{p}_{\mathbf{2}}$ is either a satisfying assignment or a $\sim \operatorname{lit}\left(x_{\mathrm{i}}\right)$-boundary point.

Proof. Reformulating the proposition, one needs to show that $\operatorname{Unsat}\left(\boldsymbol{p}_{2}, F\right)$ is either empty or contains only clauses with $\sim \operatorname{lit}\left(x_{\mathrm{i}}\right)$. Assume that contrary, i.e. $\operatorname{Unsat}\left(\boldsymbol{p}_{\mathbf{2}}, F\right)$ contains a clause $C$ with no literal of $x_{\mathrm{i}}$. (All clauses with $\operatorname{lit}\left(x_{\mathrm{i}}\right)$ are satisfied by $\boldsymbol{p}_{2}$.) Then $C$ is falsified by $\boldsymbol{p}_{1}$ too and so $\boldsymbol{p}_{\mathbf{1}}$ is not a $\operatorname{lit}\left(x_{\mathrm{i}}\right)$-boundary point. A contradiction.

Definition 8. Proposition 3 means that for unsatisfiable formulas every $x_{\mathrm{i}}$-boundary point has the corresponding $\sim x_{\mathrm{i}}$-boundary point (and vice versa). We will call such a pair of points twin boundary points in variable $x_{\mathrm{i}}$.

Example 2. The point $\boldsymbol{p}_{\mathbf{2}}=\left(x_{1}=0, x_{2}=1, x_{3}=1\right)$ of Example 1 is an $\sim x_{3}$-boundary point. The point $\boldsymbol{p}_{\mathbf{3}}=\left(x_{1}=0, x_{2}=1, x_{3}=0\right)$ obtained from $\boldsymbol{p}_{\mathbf{2}}$ by flipping the value of $x_{3}$ falsifies only clause $C_{2}=\sim x_{2} \vee x_{3}$. So $p_{3}$ is an $x_{3}$-boundary point.

\subsection{Elimination of Boundary Points by Adding Resolvents}

In this subsection, we prove the following propositions. Clauses of a CNF formula $F$ falsified by twin boundary points can be resolved (Proposition 4). Adding such a resolvent to $F$ eliminates these boundary points (Proposition 5). Adding the resolvents of a resolution proof eventually eliminates all boundary points (Proposition 6). A lit $\left(x_{\mathrm{i}}\right)$-boundary point can be eliminated only by a resolution on variable $x_{\mathrm{i}}$ (Proposition 7). If formula $F$ has a $\operatorname{lit}\left(x_{\mathrm{i}}\right)$-boundary point, any resolution proof that $F$ is unsatisfiable has a resolution on variable $x_{\mathrm{i}}$. (Proposition 8).

Definition 9. Let $C_{1}$ and $C_{2}$ be two clauses that have opposite literals of variable $x_{\mathrm{i}}$ (and no opposite literals of any other variable). The resolvent $C$ of $C_{1}$ and $C_{2}$ is the clause consisting of all the literals of $C_{1}$ and $C_{2}$ but the literals of $x_{\mathrm{i}}$. The clause $C$ is said to be obtained by a resolution operation on variable $x_{\mathrm{i}} . C_{1}$ and $C_{2}$ are called the parent clauses. 
Proposition 4. Let $p_{1}$ and $p_{2}$ be twin boundary points of a CNF formula $F$ in variable $x_{\mathrm{i}}$. Let $C_{1}$ and $C_{2}$ be two arbitrary clauses falsified by $\boldsymbol{p}_{1}$ and $\boldsymbol{p}_{2}$ respectively. Then a) $C_{1}, C_{2}$ can be resolved on variable $x_{\mathrm{i}}$; b) $C\left(\boldsymbol{p}_{1}\right)=0, C\left(\boldsymbol{p}_{2}\right)=0$ where $C$ is the resolvent of $C_{1}$ and $C_{2}$.

Proof. Since $C_{1}\left(\boldsymbol{p}_{1}\right)=0, C_{2}\left(\boldsymbol{p}_{2}\right)=0$ and $\boldsymbol{p}_{1}$ and $\boldsymbol{p}_{2}$ are twin boundary points in $x_{\mathrm{i}}, C_{1}$ and $C_{2}$ have opposite literals of variable $x_{\mathrm{i}}$. Since $\boldsymbol{p}_{\mathbf{1}}$ and $\boldsymbol{p}_{\mathbf{2}}$ are different only in the value of $x_{\mathrm{i}}$, clauses $C_{1}$ and $C_{2}$ can not contain opposite literals of a variable other than $x_{\mathrm{i}}$. (Otherwise, $\boldsymbol{p}_{\mathbf{1}}$ and $\boldsymbol{p}_{\mathbf{2}}$ had to be different in values of at least 2 variables.) Since $\boldsymbol{p}_{\mathbf{1}}$ and $\boldsymbol{p}_{\mathbf{2}}$ are different only in the value of $x_{\mathrm{i}}$, they both set to 0 all the literals of $C_{1}$ and $C_{2}$ but literals of $x_{\mathrm{i}}$. So the resolvent $C$ of $C_{1}$ and $C_{2}$ is falsified by $\boldsymbol{p}_{\mathbf{1}}$ and $\boldsymbol{p}_{2}$.

Example 3. Points $\boldsymbol{p}_{2}=\left(x_{1}=0, x_{2}=1, x_{3}=1\right)$ and $\boldsymbol{p}_{3}=\left(x_{1}=0, x_{2}=1, x_{3}=0\right)$ from Examples 1 and 2 are twin boundary points in variable $x_{3}$. Unsat $\left(\boldsymbol{p}_{2}, F\right)=\left\{C_{4}, C_{5}\right\}$ and $\operatorname{Unsat}\left(\boldsymbol{p}_{3}, F\right)=\left\{C_{2}\right\}$. For example, $C_{4}=x_{1} \vee \sim x_{3}$, can be resolved with $C_{2}=\sim x_{2} \vee x_{3}$ on variable $x_{3}$. Their resolvent $C=x_{1} \vee \sim x_{2}$ is falsified by both $\boldsymbol{p}_{2}$ and $\boldsymbol{p}_{3}$.

Proposition 5. Let $\boldsymbol{p}_{\mathbf{1}}$ and $\boldsymbol{p}_{\mathbf{2}}$ be twin boundary points in variable $x_{\mathrm{i}}$ and $C_{1}$ and $C_{2}$ be clauses falsified by $\boldsymbol{p}_{1}$ and $\boldsymbol{p}_{2}$ respectively Then adding the resolvent $C$ of $C_{1}$ and $C_{2}$ to $F$ eliminates the boundary points $\boldsymbol{p}_{\mathbf{1}}$ and $\boldsymbol{p}_{\mathbf{2}}$. That is pairs $\left(x_{\mathrm{i}}, \boldsymbol{p}_{\mathbf{1}}\right)$ and $\left(\sim x_{\mathrm{i}}, \boldsymbol{p}_{2}\right)$ are not in the set Bnd_pnts $\left(F \wedge C\right.$ ) ( here we assume that $\boldsymbol{p}_{\mathbf{1}}$ is an $x_{\mathrm{i}}$-boundary point and $\boldsymbol{p}_{\mathbf{2}}$ is a $\sim x_{\mathrm{i}}$-boundary point of $F$ ).

Proof. According to Proposition 4, any clauses $C_{1}$ and $C_{2}$ falsified by $\boldsymbol{p}_{\mathbf{1}}$ and $\boldsymbol{p}_{\mathbf{2}}$ respectively can be resolved in $x_{\mathrm{i}}$ and $\boldsymbol{p}_{1}$ and $\boldsymbol{p}_{2}$ falsify the resolvent $C$ of $C_{1}$ and $C_{2}$. Since clause $C$ does not have a literal of $x_{\mathrm{i}}, \boldsymbol{p}_{\mathbf{1}}$ is not an $x_{\mathrm{i}}$-boundary point and $\boldsymbol{p}_{\mathbf{2}}$ is not a $\sim x_{\mathrm{i}}$-boundary point of $F \wedge C$.

Proposition 6. If a CNF formula $F$ contains an empty clause, then $B n d p h t s(F)=\varnothing$. Proof. For any complete assignment $\boldsymbol{p}$, the set $\operatorname{Unsat}(\boldsymbol{p}, F)$ contains the empty clause of $F$. So $\boldsymbol{p}$ can not be a $\operatorname{lit}\left(x_{\mathrm{i}}\right)$-boundary point.

Proposition 6 works only in one direction, i.e. if $\operatorname{Bnd} \operatorname{pnts}(F)=\varnothing$, it does not mean that $F$ contains an empty clause. Proposition $\overline{6}$ implies that, given an unsatisfiable formula $F$ for which $B n d \operatorname{pnts}(F)$ is not empty, the resovlents of any resolution proof of unsatisfiability of $F$ eventually eliminate all the boundary points.

Proposition 7. Let $F$ be a CNF formula and $\boldsymbol{p}$ be a $\operatorname{lit}\left(x_{\mathrm{i}}\right)$-boundary point of $F$. Let $C$ be the resolvent of clauses $C_{1}$ and $C_{2}$ of $F$ that eliminates $\boldsymbol{p}$ (i.e. $\left(\operatorname{lit}\left(x_{\mathrm{i}}\right), \boldsymbol{p}\right)$ is not in Bnd_pnts $(F \wedge C))$. Then $C$ is obtained by resolution on variable $x_{\mathrm{i}}$. In other words, a $\operatorname{lit}\left(x_{\mathrm{i}}\right)$-boundary point can be eliminated only by adding to $F$ a resolvent on variable $x_{\mathrm{i}}$. Proof. Assume the contrary i.e. adding $C$ to $F$ eliminates $\boldsymbol{p}$ and $C$ is obtained by resolving $C_{1}$ and $C_{2}$ on variable $x_{\mathrm{j}}, j \neq i$. Since $C$ eliminates $\boldsymbol{p}$ as a $\operatorname{lit}\left(x_{\mathrm{i}}\right)$-boundary point, it is falsified by $\boldsymbol{p}$ and does not contain $\operatorname{lit}\left(x_{\mathrm{i}}\right)$. This means that neither $C_{1}$ nor $C_{2}$ contain variable $x_{\mathrm{i}}$. Since $C$ is falsified by $\boldsymbol{p}$, one of the parent clauses, say clause $C_{1}$, is falsified by $\boldsymbol{p}$ too. Since $C_{1}$ does not contain literal $\operatorname{lit}\left(x_{\mathrm{i}}\right), \boldsymbol{p}$ is not a $\operatorname{lit}\left(x_{\mathrm{i}}\right)$ boundary point of $F$. A contradiction. 
Proposition 8. Let $\boldsymbol{p}$ be a $\operatorname{lit}\left(x_{\mathrm{i}}\right)$-boundary point of a CNF formula $F$. Then any resolution derivation of an empty clause from $F$ has to contain a resolution operation on variable $x_{\mathrm{i}}$.

Proof. According to Proposition 6, every boundary point of $F$ is eventually eliminated in a resolution proof. According to Proposition 7, a $\operatorname{lit}\left(x_{\mathrm{i}}\right)$-boundary point can be eliminated only by adding to $F$ a clause produced by resolution on variable $x_{\mathrm{i}}$.

\subsection{Boundary Points and Clause Redundancy}

In this subsection, we show that redundant clauses (e.g. conflict clauses) can be used in resolutions as parent clauses to eliminate boundary points.

Definition 10. A clause $C$ of a CNF formula $F$ is called redundant if $F \backslash\{C\} \rightarrow C$. Proposition 9. Let $C$ be a clause of a CNF formula $F$. Let $\operatorname{lit}\left(x_{\mathrm{i}}\right)$ be a literal of $C$. Suppose that no $\operatorname{lit}\left(x_{\mathrm{i}}\right)$-boundary point of $F$ falsifies clause $C$. Then $C$ is redundant.

Proof. Assume the contrary, i.e. $C$ is not redundant. Then there is an assignment $\boldsymbol{p}$ such that $C$ is falsified and all the other clauses of $F$ are satisfied. Then $p$ is a $\operatorname{lit}\left(x_{\mathrm{i}}\right)$ boundary point. A contradiction.

Importantly, Proposition 9 works only in one direction. That is the fact that a clause $C$ is redundant in $F$ does not mean that no boundary point of $F$ falsifies $C$. Let CNF formula $F\left(x_{1}, x_{2}\right)$ consist of four clauses: $\sim x_{1}, x_{1}, x_{1} \vee \sim x_{2}, x_{1} \vee x_{2}$. Although the clause $x_{1}$ is redundant in $F, \boldsymbol{p}=\left(x_{1}=0, x_{2}=0\right)$ is an $x_{1}$-boundary point falsifying $x_{1}$ (and $x_{1} \vee x_{2}$ ). The resolvent of clauses $x_{1}$ and $\sim x_{1}$ eliminates $\boldsymbol{p}$ as a boundary point.

\section{Resolution Proofs and Boundary Points}

In this section, we view construction of a resolution proof as a process of boundary point elimination and give a metric for measuring potential proof redundancy.

\subsection{Resolution Proof as Boundary Point Elimination}

First, we define the notion of a resolution proof [3] and a boundary resolution.

Definition 11. Let $F$ be an unsatisfiable formula. Let $R_{1}, \ldots, R_{\mathrm{k}}$ be a set of clauses such that a) each clause $R_{\mathrm{i}}$ is obtained by resolution operation where a parent clause is either a clause of $F$ or the resolvent of a previous resolution operation; b) clauses $R_{\mathrm{i}}$ are numbered in the order they are derived; c) $R_{\mathrm{k}}$ is an empty clause; Then the set of resolutions that produced the resolvents $R_{1}, . ., R_{\mathrm{k}}$ is called a resolution proof. We assume that this proof is irredundant i.e. removal of any non-empty subset of these $k$ resolvents breaks condition a).

Definition 12. Let $R_{1}, . ., R_{\mathrm{k}}$ be the set of resolvents forming a resolution proof that a CNF formula $F$ is unsatisfiable. Denote by $F_{\mathrm{i}}$ the CNF formula that is equal to $F$ for $i=1$ and to $F \cup\left\{R_{1}, . ., R_{\mathrm{i}-1}\right\}$ for $i=2, \ldots, k$. In other words, $F_{\mathrm{i}}$ consists of the initial clauses of $F$ and first $i-1$ resolvents. We will say that the $i$-th resolution (i.e. one that produces resolvent $R_{\mathrm{i}}$ ) is non-boundary if Bnd_pnts $\left(F_{\mathrm{i}}\right)=\operatorname{Bnd}$ phnts $\left(F_{\mathrm{i}+1}\right)$. Otherwise 
(i.e. if Bnd pnts $\left(F_{\mathrm{i}}\right) \supset$ Bnd pnts $\left(F_{\mathrm{i}+1}\right)$, because adding a clause can not create a boundary point), $i$-th resolution is called boundary. So a resolution operation is boundary if adding $R_{\mathrm{i}}$ to $F_{\mathrm{i}}$ eliminates a boundary point.

In the previous section, we showed that eventually all the boundary points of a $\mathrm{CNF}$ formula $F$ are removed by resolvents. Importantly, a lit $\left(x_{\mathrm{i}}\right)$-boundary point mandates a resolution on variable $x_{\mathrm{i}}$. Besides, as we showed in Section 3.3. even redundant clauses can be used to produce new resolvents eliminating boundary points. It is important because, all clauses derived by resolution (in particular conflict clauses generated by modern SAT-solvers) are redundant. So the derived clauses are as good for boundary point elimination as the original ones.

A natural question arises about the role of non-boundary resolutions. When answering this question it makes sense to separate redundant and irredundant formulas. (A CNF formula $F$ is said to be irredundant if no clause of $F$ is redundant, see Definition 10) For a redundant formula, one may have to use non-boundary resolutions. (In particular, a heavily redundant formula may not have boundary points at all. In such a case, every resolution operation is non-boundary.) For irredundant formulas the situation is different.

Proposition 10. Let $F$ be an irredundant formula of $m$ clauses. Then $F$ has at least $d$ boundary points where $d$ is the total number of literals in the clauses of $F$.

Proof. Let $C$ be a clause of $F$. Then there is a complete assignment $\boldsymbol{p}$ falsifying $C$ and satisfying the clauses of $F \backslash\{C\}$. This assignment is a lit $\left(x_{\mathrm{i}}\right)$-boundary point where $\operatorname{lit}\left(x_{\mathrm{i}}\right)$ is a literal of $C$.

As far as irredundant formulas are concerned some natural questions arise. Given an irredundant formula, can one always build a resolution proof using only boundary resolutions? If so, what is the relation between such a limited proof system and general resolution? Can general resolution produce proofs shorter than in this limited proof system?

We do not answer the questions above theoretically. Instead we introduce a metric measuring the Share of Boundary Resolutions (SBR-metric) to check if proof quality depends on the value of SBR metric. (This value is computed as the percent of boundary resolutions of a proof). In Section 6, we give some experimental evidence that the value of SBR metric for short specialized proofs for equivalence checking formulas is much higher than for proofs generated by a SAT-solver with conflict driven learning.

\subsection{SBR metric and Proof Redundancy}

Although we do not know the nature of non-boundary resolutions we still can argue that the low value of SBR metric may mean some proof redundancy. The reason is that such a redundancy indeed leads to the appearance of non-boundary resolutions. We give two examples of that below.

Not sharing resolutions of conflict clause derivations. In a typical SAT-solver with conflict-driven learning, the only type of clauses learned are conflict clauses (this applies to the Sat-solver DMRP-SAT that we used in our experiments). On the other hand, a conflict clause is the result of many resolution operations. The intermediate resolvents of these operations are usually discarded. It may be the case though that for 
two conflict clauses $C_{1}$ and $C_{2}$ resolution proofs of their derivation share some intermediate resolvents. When the value of SBR metric is computed, all resolvents are taken into consideration. This lack of sharing resolutions used in conflict clause derivations would lead to appearance of non-boundary resolutions (a resolution deriving a clause produced earlier by some other resolution can not eliminate a boundary point).

Appearance of unsatisfiable subformulas. Even if the initial unsatisfiable CNF formula $F$ to be solved is irredundant, an unsatisfiable subformula of $F$ inevitably appears due to the addition of new clauses. (In particular, one can view an empty clause as an unsatisfiable subformula of the final CNF formula.) Let $F_{1}$ be an unsatisfiable subformula of $F$. Let $\operatorname{Vars}(F)$ and $\operatorname{Vars}\left(F_{1}\right)$ denote the sets of variables of $F$ and $F_{1}$. Then no $\operatorname{lit}\left(x_{\mathrm{i}}\right)$-boundary point exists if $x_{\mathrm{i}}$ is in $\operatorname{Vars}(F) \backslash \operatorname{Vars}\left(F_{1}\right)$. (The set of clauses falsified by $\boldsymbol{p}$ contains at least one clause of $F_{1}$ and such a clause does not have a literal of $x_{\mathrm{i}}$.) So any resolution on a variable of $\operatorname{Vars}(F) \backslash \operatorname{Vars}\left(F_{1}\right)$ is nonboundary.

The appearance of unsatisfiable subformulas may lead to increasing the share of non-boundary resolutions in the final proof. For example, instead of deriving an empty clause from the clauses of $F_{1}$, the SAT-solver may first derive some clauses having variables of $\operatorname{Vars}\left(F_{1}\right)$ from clauses of $F \backslash F_{1}$. It is possible since clauses of $F \backslash F_{1}$ may contain variables of $\operatorname{Vars}\left(F_{1}\right)$. When deriving such clauses the SAT-solver may use (non-boundary) resolutions on variables of $\operatorname{Vars}(F) \backslash \operatorname{Vars}\left(F_{1}\right)$, which leads to redundancy of the final proof.

\section{Equivalence Checking Formulas}

In this section, we introduce the formulas we use in the experimental part of this paper. These are the formulas that describe equivalence checking of two copies of a combinational circuit. In Subsection 5.1 we show how such formulas are constructed. In Subsection 5.2. we describe how short proofs of unsatisfiability particularly tailored for equivalence checking formulas can be built.

\subsection{Building Equivalence Checking Formulas}

Let $N$ and $N^{*}$ be two single-output combinational circuits. To check their functional equivalence one constructs a circuit called a miter (we denote it as $\operatorname{Miter}\left(N, N^{*}\right)$ ). It is a circuit that is satisfiable (i.e. its output can be set to 1 ) if and only if $N$ and $N^{*}$ are not functionally equivalent. ( $N$ and $N^{*}$ are not functionally equivalent if there is an input assignment for which $N$ and $N^{*}$ produce different output values.) Then a CNF formula $F_{\text {Miter }}$ is generated that is satisfiable if and only if $\operatorname{Miter}\left(N, N^{*}\right)$ is satisfiable. In our experiments we built a miter of two identical copies of the same circuit. In such a case $\operatorname{Miter}\left(N, N^{*}\right)$ is always unsatisfiable and so is CNF formula $F_{\text {Miter. }}$.

Example 4. Figure 1 shows the miter of copies $N$ and $N^{*}$ of the same circuit. Here $g_{1}, g_{1} *$ are OR gates, $g_{2}, g_{2}{ }^{*}$ are AND gates and $h$ is an XOR gate (implementing modulo-2 sum). Note that $N$ and $N^{*}$ have the same set of input variables but different 


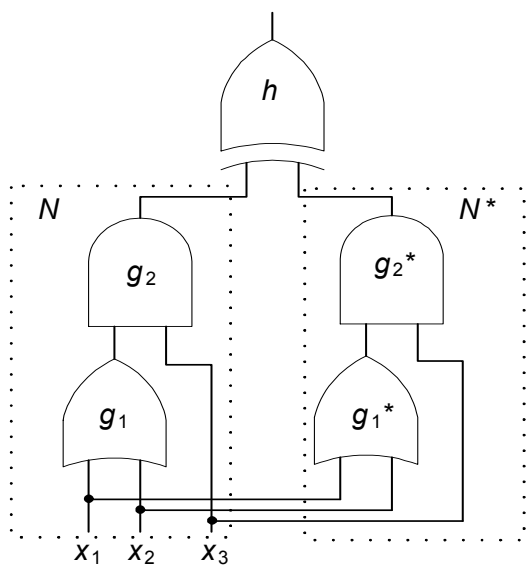

intermediate and output variables. Since $g_{2} \oplus g_{2}{ }^{*}$ evaluates to 1 if and only if $g_{2} \neq$ $g_{2}{ }^{*}$, and $N$ and $N^{*}$ are functionally equivalent, the circuit $\operatorname{Miter}\left(N, N^{*}\right)$ evaluates only to 0 .

A CNF formula $F_{\text {Miter }}$ whose satisfiability is equivalent to that of $\operatorname{Miter}\left(N, N^{*}\right)$ is formed as $F_{\mathrm{N}} \wedge F_{\mathrm{N}^{*}} \wedge F_{\text {xor }}$ $\wedge h$. Here $F_{\mathrm{N}}$ and $F_{\mathrm{N}^{*}}$ are formulas specifying the functionality of $N$ and $N^{*}$ respectively. The formula $F_{\text {xor }}$ specifies the functionality of the XOR gate $h$ and the unit clause $h$ forces the output of $\operatorname{Miter}\left(N, N^{*}\right)$ to be set to 1 .

Figure 1. Circuit $\operatorname{Miter}\left(N, N^{*}\right)$

Since, in our case, the miter evaluates only to 0 , the formula $F_{\mathrm{M}}$ is unsatisfiable.

Formulas $F_{\mathrm{N}}$ and $F_{\mathrm{N}^{*}}$ are formed as the conjunction of subformulas describing the gates of $N$ and $N^{*}$. For instance, $F_{\mathrm{N}}=F_{\mathrm{g} 1} \wedge F_{\mathrm{g} 2}$ where, for example, $F_{\mathrm{g} 1}=\left(x_{1} \vee x_{2}\right.$ $\left.\vee \sim g_{1}\right) \wedge\left(\sim x_{1} \vee g_{1}\right) \wedge\left(\sim x_{2} \vee g_{1}\right)$ specifies the functionality of an OR gate. Each clause of $F_{\mathrm{g} 1}$ rules out some inconsistent assignments to the variables of gate $g_{1}$. For example, the clause $\left(x_{1} \vee x_{2} \vee \sim g_{1}\right)$ rules out the assignment $x_{1}=0, x_{2}=0, g_{1}=1$.

\subsection{Short proofs for equivalence checking formulas}

For a CNF formula $F_{\text {Miter }}$ describing equivalence checking of two copies $N, N^{*}$ of the same circuit, there is a short resolution proof that $F_{\text {Miter }}$ is unsatisfiable. This proof is linear in the number of gates in $N$ and $N^{*}$. The idea of this proof is as follows. For every pair $g_{\mathrm{i}}, g_{\mathrm{i}}{ }^{*}$ of the corresponding gates of $N$ and $N^{*}$, the clauses of CNF formula $E q\left(g_{i}, g_{\mathrm{i}}{ }^{*}\right)$ specifying the equivalence of variables $g_{\mathrm{i}}, g_{\mathrm{i}}{ }^{*}$ are derived. Here $E q\left(g_{\mathrm{i}}, g_{\mathrm{i}}{ }^{*}\right)$ is equal to $\left(\sim g_{\mathrm{i}} \vee g_{\mathrm{i}}{ }^{*}\right) \wedge\left(g_{\mathrm{i}} \vee \sim g_{\mathrm{i}}{ }^{*}\right)$. These clauses are derived according to topological levels of gates $g_{\mathrm{i}}, g_{\mathrm{i}}{ }^{*}$ in $\operatorname{Miter}\left(N, N^{*}\right)$. (The topological level of a gate $g_{\mathrm{i}}$ is the length of the longest path from an input to gate $g_{\mathrm{i}}$ measured in the number of gates on this path.) First, clauses of $E q\left(g_{i}, g_{i}{ }^{*}\right)$ are derived for all pairs of gates $g_{\mathrm{i}}, g_{\mathrm{i}}{ }^{*}$ of topological level 1. Then using previously derived $E q\left(g_{\mathrm{i}}, g_{\mathrm{i}}{ }^{*}\right)$, same clauses are derived for the pairs of gates $g_{\mathrm{j}}, g_{\mathrm{j}}{ }^{*}$ of topological level 2 and so on.

Eventually, the clauses of $E q\left(g_{\mathrm{s}}, g_{\mathrm{s}}{ }^{*}\right)$ relating the output variables $g_{\mathrm{s}}, g_{\mathrm{s}}{ }^{*}$ of $N$ and $N^{*}$ are derived. Resolving the clauses of $E q\left(g_{\mathrm{s}}, g_{\mathrm{s}}{ }^{*}\right)$ and the clauses describing the XOR gate, the clause $\sim h$ is derived. Resolution of $\sim h$ and the unit clause $h$ of $F_{\text {Miter }}$ produces an empty clause.

Example 5. Let us explain the construction of the proof using the CNF $F_{\text {Miter }}$ from Example 4. Gates $g_{1}, g_{1}$ * have topological level 1 in $\operatorname{Miter}\left(N, N^{*}\right)$. So first, the clauses of $E q\left(g_{1}, g_{1}{ }^{*}\right)$ are obtained. They are derived from the CNF formulas $F_{\mathrm{g} 1}$ and $F_{\mathrm{g} 1 *}$ describing gates $g_{1}$ and $g_{1}{ }^{*}$. That the clauses of $E q\left(g_{1}, g_{1}{ }^{*}\right)$ can be derived from $F_{\mathrm{g} 1} \wedge$ $F_{\mathrm{g} 1} *$ just follows from the completeness of resolution and the fact that $E q\left(g_{1}, g_{1}{ }^{*}\right)$ is 
implied by the CNF formula $F_{\mathrm{g} 1} \wedge F_{\mathrm{g}^{*}}$. (This implication is due to the fact that $F_{\mathrm{g} 1}$ and $F_{\mathrm{g} 1} *$ describe two functionally equivalent gates with the same set of input variables). More specifically, the clause $\sim g_{1} \vee g_{1}$ * is obtained by resolving the clause $x_{1} \vee x_{2} \vee \sim g_{1}$ of $F_{\mathrm{g} 1}$ with the clause $\sim x_{1} \vee g_{1^{*}}$ of $F_{\mathrm{g} 1}{ }^{*}$ and then resolving the resolvent with the clause $\sim x_{2} \vee g_{1}{ }^{*}$ of $F_{\mathrm{gl}^{*}}$. In a similar manner, the clause $g_{1} \vee \sim g_{1} *$ is derived by resolving the clause $x_{1} \vee x_{2} \vee \sim g_{1}{ }^{*}$ of $F_{\mathrm{g} 1 *}$ with clauses $\sim x_{1} \vee g_{1}$ and $\sim x_{2} \vee g_{1}$ of $F_{\mathrm{g} 1}$.

Then the clauses of $E q\left(g_{2}, g_{2} *\right)$ are derived (gates $g_{2}, g_{2}{ }^{*}$ have topological level 2). $E q\left(g_{2}, g_{2}{ }^{*}\right)$ is implied by $F_{\mathrm{g} 2} \wedge F_{\mathrm{g} 2} * \wedge E q\left(g_{1}, g_{1}{ }^{*}\right)$. Indeed, $g_{2}$ and $g_{2}{ }^{*}$ are functionally equivalent gates that have the same input variable $x_{3}$. The other input variables $g_{1}$ and $g_{1}{ }^{*}$ are identical too due to the presence of $E q\left(g_{1}, g_{1}{ }^{*}\right)$. So the clauses of $E q\left(g_{2}, g_{2}{ }^{*}\right)$ can be derived from clauses of $F_{\mathrm{g} 2} \wedge F_{\mathrm{g} 2} * \wedge E q\left(g_{1}, g_{1}{ }^{*}\right)$ by resolution. Then the clause $\sim h$ is derived as implied by $F_{\text {xor }} \wedge E q\left(g_{2}, g_{2^{*}}\right)$ (an XOR gate produces output 0 when its input variables have equal values). Resolution of $h$ and $\sim h$ produces an empty clause.

\section{Experimental Results}

The goal of experiments was to compare the values of SBR metric for two kinds of proofs of different quality. In the experiments we used formulas describing the equivalence checking of two copies of combinational circuits. The reason for using such formulas is that one can easily generate high-quality specialized proofs of their unsatisfiability (see Section 5). In the experiments we compared these short proofs with ones generated by the SAT-solver DMRP-SAT [11].

We performed the experiments on a Pentium-4 PC with clock frequency of $3 \mathrm{GHz}$. The CNF formulas and proofs of both types we used in the experiments can be downloaded from http://eigold.tripod.com/exper_sat_2009.tar.gz. The time limit in all experiments was set to 1 hour.

Given a resolution proof $R$ of $k$ resolutions that a CNF formula $F$ is unsatisfiable, computing the value of SBR metric of $R$ reduces to $k$ SAT-checks. In our experiments these SAT-checks were performed by a version of DMRP-SAT. Let $F_{\mathrm{i}}$ be the CNF formula $F \cup\left\{R_{1}, . ., R_{\mathrm{i}-1}\right\}$ where $R_{1}, . ., R_{\mathrm{i}-1}$ are the resolvents generated in the first $i-1$ resolutions. Let $C_{1}$ and $C_{2}$ be the clauses of $F_{\mathrm{i}}$ that are the parent clauses of the resolvent $R_{\mathrm{i}}$. Let $C_{1}$ and $C_{2}$ be resolved on variable $x_{\mathrm{j}}$. Assume that $C_{1}$ contains the positive literal of $x_{\mathrm{j}}$. Checking if $i$-the resolution eliminates an $x_{\mathrm{j}}$-boundary point can be performed as follows. First, all the clauses with a literal of $x_{\mathrm{j}}$ are removed from $F_{\mathrm{i}}$. Then one adds to $F_{\mathrm{i}}$ the unit clauses that force the assignments setting all the literals of $C_{1}$ and all the literals of $C_{2}$ but the literal $\sim x_{\mathrm{j}}$ to 0 . Denote the resulting CNF formula by $G_{\mathrm{i}}$.

If $G_{\mathrm{i}}$ is satisfiable then there is a complete assignment $\boldsymbol{p}$ that is falsified by $C_{1}$ and maybe by some other clauses with literal $x_{\mathrm{j}}$. So $\boldsymbol{p}$ is an $x_{\mathrm{j}}$-boundary point of $F_{\mathrm{i}}$. Since $\boldsymbol{p}$ falsifies all the literals of $C_{2}$ but $\sim x_{\mathrm{j}}$, it is falsified by the resolvent of $C_{1}$ and $C_{2}$. So the satisfiability of $G_{\mathrm{i}}$ means that $i$-th resolution eliminates $\boldsymbol{p}$ and so this resolution is boundary. If $G_{\mathrm{i}}$ is unsatisfiable, then no $x_{\mathrm{j}}$-boundary point is eliminated by $i$-th resolution. All boundary points come in pairs (see Proposition 3 ). So no $\sim x_{\mathrm{j}}$-boundary 
point is eliminated by $i$-th resolution either. Hence the unsatisfiability of $G_{\mathrm{i}}$ means that the $i$-th resolution is non-boundary.

Table 1. shows the value of SBR metric for the short specialized proofs. The first column gives the name of the benchmark circuit whose self-equivalence is described by the corresponding CNF formula. The size of this CNF formula is given in the second and third columns. The fourth column of Table 1 gives the size of the proof (in the number of resolutions). The fifth column shows the value of SBR metric and the last column of Table 1 gives the run time of computing this value. These run times can be significantly improved if one uses a faster SAT-solver and tunes it to solving the particular problem of computing the value of SBR metric. (For example, one can try to share conflict clauses learned in different SAT-checks.)

Looking at Table 1 one can conclude that the specialized proofs have a very high value of SBR-metric (almost every resolution operation eliminates a boundary point). The only exception is the dalu formula (84\%). The fact that the value of SBR metric for dalu and some other formulas is different from $100 \%$ is probably due to the fact that the corresponding circuits have some redundancies. Such redundancies would lead to redundancy of CNF formulas specifying the corresponding miters, which would lower the value of SBR metric.

Table 1. Computing value of SBR metric for short specialized proofs

\begin{tabular}{|l|l|l|l|l|l|}
\hline Name & \#vars & \#clauses & $\begin{array}{l}\text { \#resolu- } \\
\text { tions }\end{array}$ & $\begin{array}{l}\text { \#boundary } \\
\text { resol. \% }\end{array}$ & $\begin{array}{l}\text { run } \\
\text { time } \\
(\mathrm{s})\end{array}$ \\
\hline c432 & 480 & 1,333 & 1,569 & 95 & 10.2 \\
\hline 9symml & 480 & 1,413 & 1,436 & 100 & 4.5 \\
\hline c880 & 807 & 2,264 & 2,469 & 100 & 24.6 \\
\hline alu4 & 2,369 & 7,066 & 8,229 & 96 & 270 \\
\hline c3540 & 2,625 & 7,746 & 9,241 & 97 & 1,743 \\
\hline x1 & 4,381 & 12,991 & 12,885 & 97 & 2,890 \\
\hline dalu & 4,714 & 13,916 & 15,593 & 84 & 2,202 \\
\hline c6288 & 4,771 & 14,278 & 17,925 & 100 & 2,462 \\
\hline
\end{tabular}

Table 2. Indirect comparison of proofs generated by Minisat and DMRP-SAT

\begin{tabular}{|l|l|l|l|l|}
\hline \multirow{2}{*}{ Name } & \multicolumn{2}{|l|}{ Minisat (v1.14) } & \multicolumn{2}{l|}{ DMRP-SAT } \\
\cline { 2 - 5 } & $\begin{array}{l}\text { \#confl. } \\
\text { clauses }\end{array}$ & $\begin{array}{l}\text { run time } \\
\text { (sec.) }\end{array}$ & $\begin{array}{l}\text { \#confl. } \\
\text { clauses }\end{array}$ & $\begin{array}{l}\text { run time } \\
\text { (sec.) }\end{array}$ \\
\hline c432 & 809 & 0.05 & 374 & 0.08 \\
\hline 9symml & 259 & 0.03 & 275 & 0.08 \\
\hline c880 & 6,000 & 0.29 & 1,309 & 0.27 \\
\hline alu4 & 2,355 & 0.33 & 1,320 & 1.20 \\
\hline c3540 & 22,214 & 2.65 & 10,021 & 7.75 \\
\hline x1 & 3,294 & 0.45 & 765 & 1.06 \\
\hline dalu & 4,402 & 0.94 & 3,351 & 4.39 \\
\hline c6288 & $*$ & $>3,600$ & $*$ & $>3,600$ \\
\hline
\end{tabular}


Table 2 is meant to show that the proofs generated by DMRP-SAT have high quality (for a SAT-solver with conflict driven learning). Here we compare the proofs generated by Minisat (version 1.14) and DMRP-SAT in the number of conflict clauses. (Minisat does not generate proofs so we could not compare the actual proof sizes). Although this is an indirect comparison, it gives an idea of the quality of proofs generated by DMRP-SAT. For self-equivalence of a 16-bit multiplier (instance C6288), neither SAT-solver finished the formula within the time limit.

The values of SBR-metric for the proofs generated by DMRP-SAT are given in Table 3. The second column gives the size of resolution proofs generated by DMRPSAT. When computing the size of these proofs we removed the obvious redundancies. Namely, the derivation of the conflict clauses that did not contribute to the derivation of an empty clause was ignored. The third column shows the value of SBR metric and the last column gives the run time of computing this value. In the case the computation did not finish within the time limit, the number in parentheses shows the percent of the resolution operations processed before the computation terminated.

Table 3 shows that the size of the proofs generated by DMRP-SAT is much larger than that of specialized proofs (Table 1, fourth column). The only exception is the instance $x l$ where the two kinds of proofs have comparable size. Interestingly, $x l$ is the instance with the highest value of SBR metric (88\%) among DMRP-SAT proofs. For the rest of the formulas the value of SBR metric is much smaller. Importantly, the value of SBR metric we give for the formulas for which computation was terminated due to exceeding the time limit is higher than it should be. Typically, the later a resolution occurs in a resolution proof, the more likely it is that this resolution is non-boundary. So the early termination of SBR metric computation ignored resolutions with the highest chances to be non-boundary.

Table 3. Computing value of SBR metric for proofs generated by DMRP-SAT

\begin{tabular}{|l|l|l|l|}
\hline Name & $\begin{array}{l}\text { \#resolu- } \\
\text { tions }\end{array}$ & $\begin{array}{l}\text { \#boundary } \\
\text { resol. \% }\end{array}$ & $\begin{array}{l}\text { run time } \\
(\mathrm{s})(\% \text { of proof finished })\end{array}$ \\
\hline c432 & 7,655 & 37 & 48 \\
\hline 9 symml & 5,317 & 57 & 23 \\
\hline c880 & 24,478 & 37 & 503 \\
\hline alu4 & 98,600 & 36 & $>3,600(49 \%)$ \\
\hline c3540 & 347,264 & 54 & $>3,600(6 \%)$ \\
\hline x1 & 16,259 & 88 & 864 \\
\hline dalu & 119,553 & 40 & $>3,600(33.4 \%)$ \\
\hline
\end{tabular}

Summing up, one can conclude that for the formulas we considered in experiments, the proofs of poorer quality (generated by DMRP-SAT) have lower values of SBR metric. It remains to be seen though whether it means that these proofs are redundant in some way and so can be optimized. 


\section{Some Background}

The notion of boundary points was introduced in [12] where they were called essential points. (We decided to switch to the term "boundary point" as more precise.) Boundary points were used in [12] to help a SAT-solver prune the search space. If the subspace $x_{\mathrm{i}}=0$ does not contain a satisfying assignment or an $x_{\mathrm{i}^{-}}$ boundary point, one can claim that the symmetric subspace $x_{\mathrm{i}}=1$ can not contain a satisfying assignment either (due to Proposition 3). The same idea of search pruning was independently described in [17] and implemented in the SAT-solver Jerusat. The ideas of search pruning introduced in [12] were further developed in [2].

In [14] we formulate two proof systems meant for exploring the 1-neighborhood of clauses of the formula to be solved. The union of the 1-neighborhhoods of these clauses is essentially a superset approximation of the set of boundary points. To prove that a formula is unsatisfiable it is sufficient to eliminate all boundary points (Proposition 2). The proof systems of [14] show that one can eliminate all boundary points without generation of an empty clause. So resolution can be viewed as a special case of boundary point elimination.

The results of the present paper can also be considered as an approach to improving automatizability of resolution [6]. General resolution is most likely nonautomatizable [1]. This means that finding short proofs can not be done efficiently in general resolution. A natural way to mitigate this problem is to look for restricted versions of general resolution that are "more automatizable" i.e. that facilitate finding good proofs. Intuitively, boundary resolutions is a tiny part of the set of all possible resolutions. So the restriction of resolutions to boundary ones can be viewed as a way to make it easier to find good proofs (assuming that such a restriction does not kill all high-quality proofs.)

\section{Conclusions and Directions for Future Research}

We show that a resolution proof can be viewed as the process of boundary point elimination. We introduce the SBR metric that is the percent of resolutions of the proof that eliminate boundary points (boundary resolutions). This metric can be used for estimating proof redundancy. We experimentally show that short specialized proofs for equivalence checking formulas have high values of SBR metric. On the other hand, values of this metric for proofs generated by a SAT-solver with conflict driven learning are low. This implies that the proofs generated by this SAT-solver may have some redundancies.

The idea of treating resolution as boundary proof elimination has many interesting directions for research. Here are a few of them.

1) Testing further the conjecture that SBR metric relates to proof redundancy.

2) Proving completeness of resolution performing only boundary resolutions for irredundant CNF formulas

3) Answering the question about the nature of non-boundary resolutions. In particular, is the resolution proof system where only boundary resolutions are allowed less powerful than general resolution? 
4) Designing SAT-solvers that generate proofs with high value of SBR metric.

\section{References}

1. Alekhnovich, M., Razborov, A.: Resolution Is Not Automatizable Unless W[P] Is Tractable. SIAM J. Comput., vol. 38, no.4, pp. 1347--1363 (2008).

2. Babic, D., Bihgham, J.,Hu A.: Efficient SAT solving: beyond supercubes. In: DAC-2005, pp. 744--459.

3. Bachmair, L.,Ganzinger, H.: Resolution theorem proving. In: Robinson, A., Voronkov, A. (eds). Handbook of automated reasoning. Chap. 2, vol. 1,pp. 19-99. Elsevier Science Publ. (2001).

4. Bierre, A.: PicoSAT essentials. JSAT 4, 75--97 (2008).

5. Bierre, A.,Cimatti, A.,Clarke, Fujita M., Zhu Y.: Symbolic model checking using SAT procedures instead of BDDs. In: DAC-1999, pp. 317--320.

6. Bonet, M. L., Pitassi, T., and Raz, R: On Interpolation and Automatization for Frege Systems. SIAM J. Comput. Vol. 29, no. 6 pp. 1939--1967 (2000).

7. Clarke, E., Gupta, A.,Strichman, O.: SAT-based counterexample-guided abstraction refinement, IEEE Transactions on Computer-Aided Design of Integrated Circuits and Systems, vol. 23, No. 7, pp. 1113--1123 (2004).

8. Davis, M., Longemann, G., Loveland, D.: A Machine program for theorem proving. Communications of the ACM 5, 394--397 (1962).

9. Een, N., Sorensson, N. : An extensible SAT-solver. In: SAT-2003, LNCS, vol. 2919, pp.503--518.

10. Goldberg, E.: On Bridging Simulation and Formal Verification. In: VMCAI2008, pp.127--141.

11. Goldberg, E.: A Decision-Making Procedure for Resolution-Based SAT-Solvers. In: SAT-2008, pp. 119--132.

12. Goldberg, E., Prasad, M.,Brayton, R.: Using Problem Symmetry in Search Based Satisfiability Algorithms. In: DATE-2002, pp. 134--141.

13. Goldberg, E., Novikov, Y.: BerkMin: a Fast and Robust SAT-Solver. In: DATE2002, pp. 142--149

14. Goldberg, E.: Proving unsatisfiability of CNFs locally. J. Autom. Reasoning, vol.28, no.5, pp. 417--434 (2002).

15. McMillan, K.: Interpolation and SAT-based model checking. In: CAV 03, LNCS, vol. 2725,pp. 1--13.

16. Moskewicz, M., Madigan, C., Zhao, Y., Zhang, L., Malik, S.: Chaff: Engineering an Efficient SAT Solver. In: DAC-2001, pp. 530--535.

17. Nadel, A., Backtrack search algorithms for propositional logic satisfiability: review and innovations. Master Thesis, the Hebrew University (2002).

18. The siege sat-solver, http://www.cs.sfu.ca/ cl/software/siege/

19. Silva,J., Sakallah, K. GRASP: A Search Algorithm for Propositional Satisfiability. IEEE Transactions of Computers 48, 506--521 (1999).

20. Zhang,H.: SATO: An efficient propositional prover. CADE-1997, pp. 272--275. 